\title{
Policy-Aware Content Reuse on the Web
}

\author{
Oshani Seneviratne, Lalana Kagal, and Tim Berners-Lee \\ MIT CSAIL, Cambridge \\ Massachusetts, USA \\ \{oshani,lkagal,timbl\}@csail.mit.edu
}

\begin{abstract}
The Web allows users to share their work very effectively leading to the rapid re-use and remixing of content on the Web including text, images, and videos. Scientific research data, social networks, blogs, photo sharing sites and other such applications known collectively as the Social Web have lots of increasingly complex information. Such information from several Web pages can be very easily aggregated, mashed up and presented in other Web pages. Content generation of this nature inevitably leads to many copyright and license violations, motivating research into effective methods to detect and prevent such violations.

This is supported by an experiment on Creative Commons (CC) attribution license violations from samples of Web pages that had at least one embedded Flickr image, which revealed that the attribution license violation rate of Flickr images on the Web is around 70-90\%. Our primary objective is to enable users to do the right thing and comply with $\mathrm{CC}$ licenses associated with Web media, instead of preventing them from doing the wrong thing or detecting violations of these licenses. As a solution, we have implemented two applications: (1) Attribution License Violations Validator, which can be used to validate users' derived work against attribution licenses of reused media and, (2) Semantic Clipboard, which provides license awareness of Web media and enables users to copy them along with the appropriate license metadata.
\end{abstract}

\section{Introduction}

Content reuse, often called 'mash-ups', have existed for as long as content has existed. Musicians routinely use other songs and tunes in their compositions. Collage art is considered to be creative, and even original, although it is composed from many different sources. Scientists routinely utilize data from different sources to conduct their experiments. However, mash-ups are a peculiarly digital phenomenon of the Web age. They are entirely a product made possible by the portable, mixable and immediate nature of digital technology. A potential legal problem arises when more than one legally encumbered content or data stream is bound together with others in the form of a mash-up. The users of the original content should remain within the bounds of the permitted use of the components comprising the mash-up. They can choose to ignore these permissions, or follow them. Either way, this creates a burden on them. Ignoring the license terms puts them in peril of breaking the law, and following them slows the creative process.

A. Bernstein et al. (Eds.): ISWC 2009, LNCS 5823, pp. 553 568, 2009.
(C) Springer-Verlag Berlin Heidelberg 2009 
Licenses or policies in general are pervasive in Web applications. They play a crucial role in enhancing security, privacy and usability of the services offered on the Web 3. In this paper we limit the 'policy awareness' to scenarios that involve content reuse. We expect the policies in this context to comprise of licenses that can be expressed semantically, that are widely deployed on a range of media, and that have a large community base. CC licenses fit this description perfectly, as they provide a very clear and a widely accepted rights expression language implemented using Semantic Web technologies [11. These licenses are both machine readable and human readable, and clearly indicate to a person, who wishes to reuse content, exactly how it should be used by expressing the accepted use, permissions, and restrictions of the content.

Popular search engines, including Google, Yahoo, and even sites such as Flickr, blip.tv, OWL Music Search and SpinXpress, have advanced search options to find CC licensed content on the Web [5|32 9|2 20 26]. However, even with these human-friendly licenses and the tools to support license discovery, license violations occur due to many reasons; Users may be ignorant as to what each of the licenses mean, or forget or be too lazy to check the license terms, or give an incorrect license which violates the original content creator's intention, or intentionally ignore the CC-license given to an original work in their own interests. Therefore, it is important that we have tools and techniques to make users aware of policies that they must follow while making the process of being license-compliant as painless as possible for the user, and make it difficult for someone to become license in-compliant either deliberately or by mistake.

In essence, the work described in this paper supports the principles of information accountability 29], policy-awareness and after-the-fact violations detection instead of relying on strict up-front enforcement. This paper is organized as follows: Section 2 gives the background and an overview of the technologies used. Section 3 outlines an experiment conducted to assess the level of CC attribution license violations on the Web using Flickr images. This experiment provided the motivation to develop tools for policy aware content reuse as described later in the paper in Section 4. Section [5] discusses the related work in this area and Section [6] discusses some future work on the tools we have developed. Finally, we conclude the paper with a summary of the contributions in Section 7.

\section{Background}

To be useful, metadata needs to have three important characteristics: it has to be easy to produce, be easily embeddable within the data they describe, and be easily readable 18 . The easiest way to produce metadata is to have it be produced automatically. Any metadata that has to be produced manually by the user usually doesn't get produced at all. The easiest way to ensure that the link between metadata and the data it describes is not broken is by embedding the former inside the latter. This way, the two travel together inseparably as a package. Finally, metadata has to be accessible easily, readable both manually as 
well as programmatically. At best, the metadata should be readable by crawlers of various search engines. Since metadata and data are traveling together, if popular search engines such as Google and Yahoo can read the metadata, by default the data become available to anyone who searches for it. RDF [23] satisfies all these criteria, has lot of community support, has been adopted widely and is a W3C recommendation.

\subsection{Inline Provenance Using Metadata}

The Extensible Metadata Platform (XMP) 31 is a technology that allows one to transfer metadata along with the content by embedding the metadata in machine readable RDF using a pre-defined set of classes and properties. This technology is widely deployed to embed licenses in free-floating multimedia content such as images, audio and video on the Web. Another format which is nearly universal when it comes to images is the Exchangeable Image File format (EXIF) [7]. The International Press Telecommunications Council (IPTC) photo metadata standard [14] is another well known standard. The metadata tags defined in these standards cover a broad spectrum of properties including date \& time information, camera settings, thumbnail for previews and more importantly, the description of the photos including the copyright information. However both EXIF and IPTC formats do not store metadata in RDF. Also, one major drawback of inline metadata formats such as XMP, EXIF and IPTC is that it is embedded in a binary file, completely opaque to nearly all users, whereas metadata expressed in RDFa 24] will require colocation of metadata with human visible HTML. In addition to that, these metadata formats can only handle limited number of properties and lack the rich expressivity required for many content reuse policies.

\subsection{Policies for Rights Enforcement on the Web}

Policies governing the reuse of digital content on the Web can take several forms. It can be upfront enforcement mechanisms such as Digital Rights Management (DRM) approaches, or rights expression mechanisms such as Creative Commons licenses where users are given the freedom to reuse content, subject to several restrictions and conditions.

When it comes to DRM, distribution and usage of copyrighted content is often controlled by up-front policy enforcement. These systems usually restrict access to the content, or prevent the content from being used within certain applications. The core concept in DRM is the use of digital licenses, which grant certain rights to the user. These rights are mainly usage rules that are defined by a range of criteria, such as frequency of access, expiration date, restriction to transfer to another playback device, etc. An example of a DRM enforcement would be a DRM enabled playback device not playing a DRM controlled media transferred from another playback device, or not playing the media after the rental period has ended. The use of DRM to express and enforce rights on content 
on the Web raises several concerns. First, consumer privacy and anonymity are compromised. Second, the authentication process in the DRM system usually requires the user to reveal her identity to access the protected content, leading to profiling of user preferences and monitoring of user activity at large [8]. Third, the usability of the content is questionable since the user is limited to using proprietary applications to view or play the digital content, producing vendor lock-in. Similarly, 'copyright notices' or 'end-user license agreements' describe the conditions of usage of copyrighted material. A user of that particular material should abide by the license that covers the usage, and if any of the conditions of usage described in that license are violated, then the original content creator has the right to take legal action against the violator.

In contrast, $\mathrm{CC}$ has been striving to provide a simple, uniform, and understandable set of licenses that content creators can issue their content under to enable reuse with much less restrictions. Often, people tend to post their content with the understanding that it will be quoted, copied, and reused. Further, they may wish that their work only be used with attribution, used only for noncommercial use, distributed with a similar license or will be allowed in other free culture media. To allow these use restrictions $\mathrm{CC}$ has composed four distinct license types: $B Y$ (attribution), $N C$ (non-commercial), $N D$ (no-derivatives) and $S A$ (share-alike) that can be used in combinations that best reflect the content creator's rights. In order to generate the license in XHTML easily, CC offers a license chooser that is hosted at http://creativecommons.org/license. With some user input about the work that is being licensed, the license chooser generates a snippet of XHTML that contains the RDFa 24] to be included when the content is published on the Web. Content creators have the flexibility to express their licensing requirements using the Creative Commons Rights Expression Language $(c c R E L) \sqrt{1}$ [1] and are not forced into choosing a pre-defined license for their works. Also, they are free to extend licenses to meet their own requirements. ccREL allows a publisher of a work to give additional permissions beyond those specified in the CC license with the use of the cc:morePermissions property to reference commercial licensing brokers or any other license deed, and $d c$ :source to reference parent works.

\section{Motivation}

Unless a particular piece of content on the Web has some strict access control policies, most users do not feel the need to check for the license it is under and be license compliant. To verify this hypothesis we conducted an experiment to assess the level of license violations on the Web. Specifically, the goal of the experiment was to obtain an estimation for the level of $\mathrm{CC}$ attribution license violations on the Web using Flickr images2.

${ }^{1}$ ccREL is the standard recommended by the Creative Commons for machine readable expressions of the meaning of a particular license.

2 As of April 2009, Flickr has over 100 million Creative Commons Licensed images. Thus it provided a large sample base for our experiment. 


\subsection{Experiment Setup}

The sampling method used for the experiment was simple random sampling on clusters of Web pages gathered during a particular time frame. To ensure a fair sample we used the Technorati blog indexer 3 without hand-picking Web pages to compose the sample to check for attribution license violations. We limited the number of Web pages to around 70, and the number of images to less than 500, so that we could do a manual inspection to see if there are any false positives, false negatives and/or any other errors. This also enabled us to check if the different samples contained the same Web pages. We found that the correlation among the samples was minimal.

Sample Collection using the Technorati API: The Technorati blog indexer crawls and indexes blog-style Web pages and keeps track of what pages link to them, what pages they link to, how popular they are, how popular the pages that link to them are, and so on. Technorati data are time dependent, and therefore the Technorati Authority Rank, a measurement that determines the top $n$ results from any query to the Technorati API, is based on the most recent activity of a particular Web pag@. The Technorati Cosmos querying functions allow the retrieval of results for blogs linking to a given base URI based on the authority rank. Therefore to generate the samples, we used the Technorati Cosmos functions by retrieving results for Web pages linking to Flickr server farm URIs that have this particular format: http://farm<farm-id $>$.static. flickr.com/<server-id $\rangle /\langle i d\rangle_{-}<$secret> . (jpg|gif $\mid$png) 5 . Since the Flickr site has several server farms, each time the experiment was run, the base URIs were randomly generated by altering the Flickr server farm-ids. In addition to that, we made sure that the samples were independent of each other and the correlation among the samples were low by running the experiment three times with two weeks between each trial. This is because the Authority Rank given to a Web page by Technorati, and hence the results returned from the Cosmos query functions dynamically changes as new content gets created.

Criteria for Checking Attribution: Flickr is still using the older CC 2.0 recommendation. Therefore, Flickr users do not have that much flexibility in specifying their own attributionURL or the attributionName as specified in ccREL. However, it is considered good practice to give attribution by linking to the Flickr user profile or by giving the Flickr user name (which could be interpreted as the attributionURL and the attributionName respectively), or at least, point

${ }^{3}$ Implemented using the Technorati API detailed at http://technorati.com/developers/api

${ }^{4}$ We expected that the use of the Technorati Authority Rank would introduce a bias in our sample. This is because the top Web pages from the Technorati blog indexer are probably well visited, hence more pressure on the Web page owners to fix errors in attribution. However, the results proved otherwise.

${ }^{5}$ According to http://www.flickr.com/services/api/misc.urls.html, all Flickr images have that particular URI pattern. 
to the original source of the image [12]. Therefore, the criteria for checking attribution consist of looking for the attributionURL or the attributionName or any source citations within a reasonable level of scoping from where the image is embedded in the Document Object Model (DOM) of the corresponding Web page.

\subsection{Results}

The results from the 3 trials are given in Fig 1 These results have misattribution and non-attribution rates ranging from $78 \%$ to $94 \%$ signaling that there is a strong need to promote license or policy awareness among reusers of content. The entire result set includes the total number of Web pages tested, number of images in all of those Web pages, number of properly attributed images, number of misattributed or non-attributed images, and the number of instances that led to an error due to parsing errors resulting from bad HTML. Using these values, the percentages of misattribution and non-attribution for each sample were calculated.
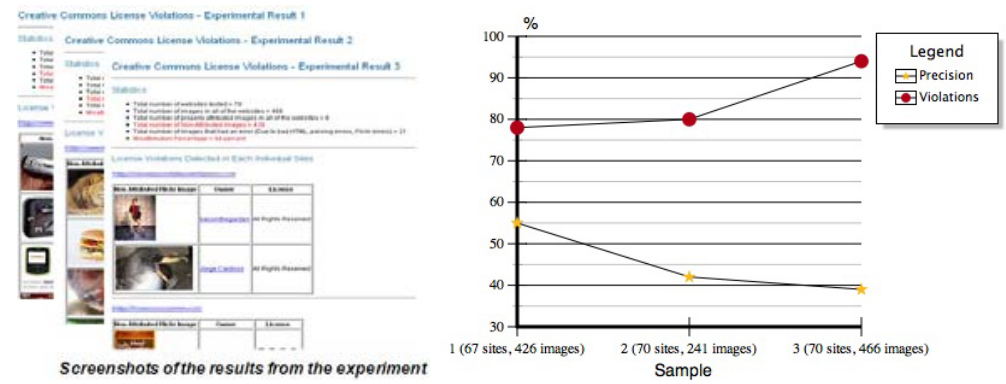

Fig. 1. Left: Screenshots of the results from the experiment (Refer to http://dig.csail.mit.edu/2008/WSRI-Exchange/results for more information). Right: Attribution violations rate and Precision obtained after correcting for self-attribution.

\subsection{Issues and Limitations of the Experiment}

As in any experiment, there are several issues and limitations in this experiment.

\section{Results include cases where the users have not attributed themselves:}

For example, consider the case where a user uploads her photos on Flickr, and uses those photos in one of her own Web pages without attributing herself. As the copyright holder of the work, she can do pretty much whatever she wants with those, even though the CC BY license deed states: "If You Distribute you must keep intact all copyright notices for the Work and provide (i) the name of the Original Author..." [4. However if she fails to include the license notice and the attribution to herself, she may be setting a precedent for the violation of her own rights in the long run. From the point of view of the experiment, it was difficult to infer the page owner from the data presented in the page. 
Even if that was possible, it is hard to make a correlation between the Flickr photo owner and the page owner. However, we manually inspected the samples to see whether the misattributed images were actually from the user or not, and flagged the ones which are definitely from the original user as false positives in the results set to obtain the precision rate. After this correction, we found the precision rate of the experiment to be between $55 \%$ to $40 \%$.

Low adoption of ccREL and Attribution Scoping: We found out that a majority of the Web pages examined in this experiment have not used ccREL in marking up attribution. Therefore, we used a heuristic to check for the existence of attribution in the pages used in the trials. This heuristic includes the attributionName constructed from the Flickr user name, or the attributionURL constructed from the Flickr user profile URI, or the original source document's URI. We expected to find the attribution information in the parent of the DOM element or in one of the neighboring DOM elements. This can be visually correlated to finding the attribution information immediately after the content that is being reused. However, since there is no strict definition from CC as to how attribution should be scoped, someone could also attribute the original content creator somewhere else in the document. However, considering that it is possible the user intended to include more than one image from the same original content creator, and by mistake failed to attribute some images, while correctly attributing all the others, we only checked attribution information within the neighboring DOM elements, and not at the document level.

Blog Aggregators such as Tumble-logs cutting down the text and favoring short form, mixed media posts over long editorial posts: Use of such blog aggregators (for example tumblr.com) is another problem in getting an accurate assessment of attribution license violations. For example, in a blog post where a photo was reused, the original owner of the photograph may have been duly attributed. But when the tumble-log pulls in the feed from that post in the original Web page and presents the aggregated content, the attribution details may be left out. This problem is difficult to circumvent because there is no standard that specifies how aggregation should take license and attribution details into consideration.

\section{Tools to Enable Policy Awareness}

As a proof of concept we developed two tools that can be used to enable policy awareness when reusing images on the Web. Even though both tools are currently limited to image reuse, it can be easily extended to support other types of media.

\subsection{Attribution License Violations Validator for Flickr Images}

When someone aggregates content from many different sources, it is inevitable that some attribution details may be accidentally forgotten. The Attribution License Violations Validator is designed to check whether the user has properly 
cited the source by giving the due attribution to the original content creator. In order to make sure that no CC license terms of the user are violated, the author can run the CC License Violations Validator and see if some sources have been left out or whether some have been misattributed.
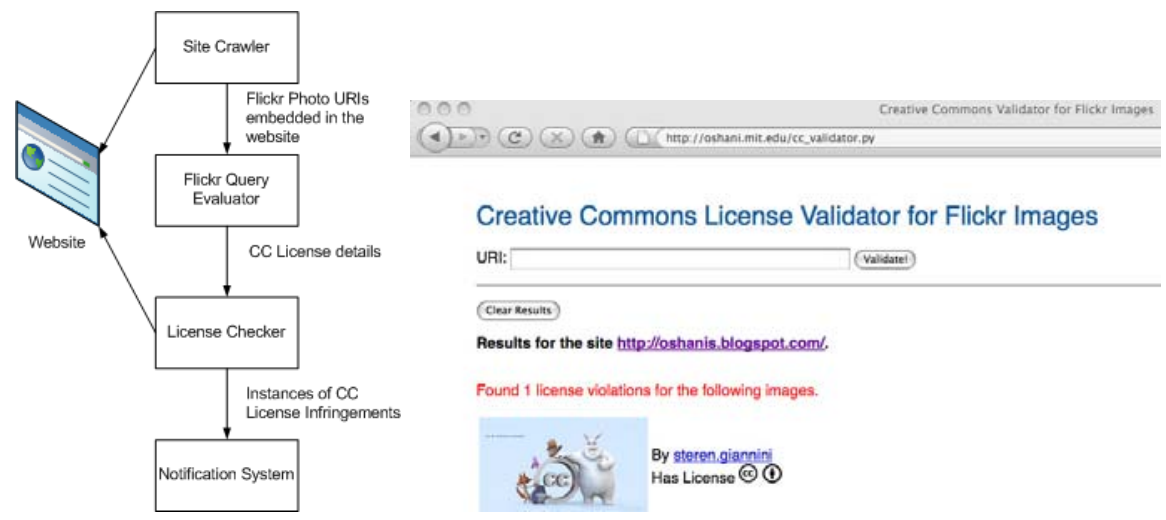

Fig. 2. Left: The Design of the Validator. Right: Output from the Validator showing the image that was not attributed properly, who the image belongs to and what license it is under.

Design and Implementation: The tool has four major components as shown in the left half of Fig 2. Once the user gives the URI where the composite work can be found, the site crawler will search for all the links embedded in the given Web page and extract any embedded Flickr photos. From each of these Flickr photo URIs, it is possible to glean the Flickr photo id. Using this photo id, all the information related to the photo is obtained by calling several methods in the Flickr API. This information includes the original creator's Flickr user account id, name, and CC license information pertaining to the photo, etc. Based on the license information of the Flickr photo, the tool checks for the attribution information that can be either the attributionName, attributionURL, source URI or any combination of those within a reasonable scoping in the containing DOM element in which the image was embedded. The 'reasonable scoping' in this case, is taken to be within the parent or the sibling nodes of the element that has the embedded image. If such information is missing, the user is presented with the details of the original content creator's name, the image along with its URI, and the license it is under, enabling the user to compose the XHTML required to properly attribute the sources used.

Challenges and Limitations: The license violations detection can only work if the image URI is actually linked from the Flickr site. Therefore if a user wants to cheat, she can easily do so by changing the image URI by uploading it to another Web space. Another complication is that a Flickr user can upload and assign CC licenses regardless of that user having the actual rights to do so. In 
other words, if someone uploads a copyrighted photo from Getty Images and assigns a CC license on Flickr, and an innocent user downloads and uses this photo, then that user will be violating the copyright law inadvertently. Therefore, we need to have some capability to track provenance of image data, and be able to identify whether a particular image has been used elsewhere in a manner that violates the original license terms. One of the major assumptions we have made in developing this tool is that attribution is specified within the parent node or the sibling nodes of the containing image element. Otherwise we classify it an instance of non-attribution. This assumption works in practice and appears to be the most logical thing to do. However, since there is no standard agreement as to what the correct scoping for attribution is, this assumption can lead to a wrong validation result. The solution to this problem is two-fold. (1) CC should give a guideline as to what the correct scoping of attribution should be relative to the content that is attributed. (2) Flickr (or any other such service) should expose the license metadata as RDF, instead of providing an API to query with. Exposing license metadata as RDF is preferred as it enables data interoperability and relieves the tool authors from having to write data wrappers for each service.

\subsection{Semantic Clipboard}

The Semantic Clipboard is a Firefox Web browser based tool integrated as part of the Tabulator, a linked data browser that can be installed as a Firefox extension 28. The primary goal of this tool is to let users reuse content with minimal effort.

Design and Implementation: The design of the Semantic Clipboard is given in Fig 3. The tool uses the 'RDFa Extractor' to glean the Creative Commons license information expressed in RDFa from the HTML page the user browses. The 'UI Enhancer' implements several menu options in the Firefox browser to select licensed images with the proper intention. The available options are given in Fig[3. For example, if a user want to see images that can be used for 'Commercial Purposes', she can select the corresponding menu item. Then the images that do not have the CC-NC clause (Creative Commons Non Commercial use) will be highlighted with an overlay on the image. The 'Attribution XHTML Constructor' is called when the user issues a copy instruction on a particular image by right-clicking on the image and selecting the context menu option 'Copy Image with License' as shown in the right half of Fig 3. Based on the license information for that particular image, the attribution XHTML snippet is constructed as specified by Creative Commons, and copied to the system clipboard. Currently two data flavors are supported: ASCII text and HTML. Therefore if the target application accepts HTML such as in a rich text editor, the source text (with the angle brackets) will not be displayed.

Challenges and Limitations: One of the hazards of combining multiple data sources is that incompatible licenses can get mixed up creating a license that basically freezes the creative process. Take for example a Non-Commercial (NC) 


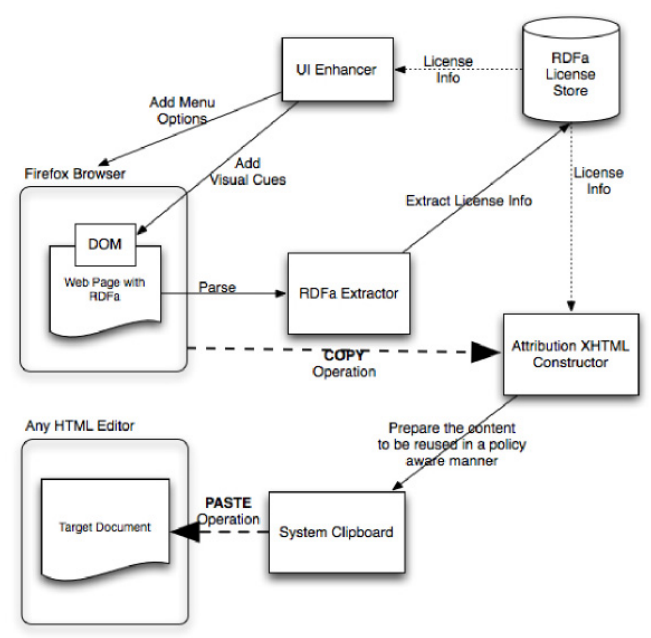

Design of the Semantic Clipboard

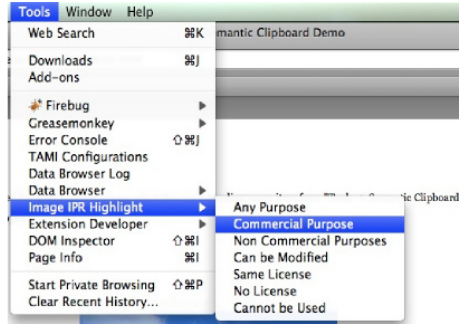

Firefox Menu

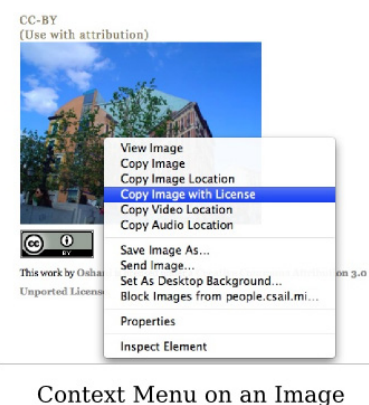

Fig. 3. Left: Semantic Clipboard Architecture. Right: Semantic Clipboard User Interface.

license that gets mixed with a Share-Alike (SA) license. An SA license requires that the resulting product be shared under exactly the same conditions as the component product under SA. The resulting license in our scenario becomes NC-SA. But while the result satisfies the first license by also being NC, it fails the second license by not being only $\mathrm{SA}$. We cannot simply ignore the $\mathrm{NC}$ clause and give the resulting work only the SA license because somebody else might use the resulting derivative work which does not have the NC clause for some commercial use violating the rights of the original creator who composed the NC component. The Semantic Clipboard does not handle such license conflicts.

\section{Related Work}

Reuse detection is important in domains such as plagiarism detection and even in biological sequence mining. Significant research has been carried out to detect reuse of text. This includes information retrieval techniques as mentioned in [17/25], where the document is treated as a sequence of symbols and substring based fingerprints are extracted from the document to determine repetitive patterns.

The CC License Syntax Validation Service 13] can be used to parse documents for embedded licenses in RDFa. After parsing the document, this service gives a list of licensed objects and each of their license authorship, version, jurisdiction, whether the license has been superseded or deprecated and whether the 
work is allowed in free cultural works, etc. However, it does not give the information as to whom the attribution should be given when reusing these license objects, like in the attribution license violations validator we have developed. In addition to that, $\mathrm{CC}$ has put much focus on coming up with ways to enable tool builders to use the CC licenses very effectively. For example, the live box on the License Deed Page as shown in Fig 4 suggests how to attribute a particular work. This is created when a CC license hyperlink that has the attributionName and the attributionURL properties to the License Deed Page is dereferenced. There are also several license aware Mozilla Firefox extensions developed by the CC. MozCC [19] is one such tool. It provides a specialized interface for displaying $\mathrm{CC}$ licenses, where the user receives visual cues when a page with RDFa metadata is encountered. This includes the display of specific CC branded icons in the browser status bar when the metadata indicates the presence of a $\mathrm{CC}$ license. However, this software does not offer the capability to copy the license attribution XHTML as in the Semantic Clipboard that we have developed.

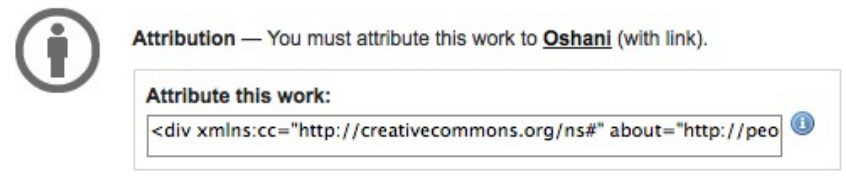

Fig. 4. CC Deed Page Displaying the Attribution XHTML

The Semantic Clipboard was actually inspired from the work done on 'XHTML Documents with Inline, Policy-Aware Provenance' [15] by Harvey Jones. Jones developed a document format that can represent information about the sources of its content, a method of excerpting from these documents that allows programs to trace the excerpt back to the source, a $\mathrm{CC}$ reasoning engine which calculates the appropriate license for the composite document, and a bookmarklet that uses all these components to recommend permissible licenses. But this tool requires all the source documents to be annotated with a special document fragment ontology, and the Paste Operation is limited to inserting copied XHTML in the top level of the document only, i.e. it does not allow copying inside existing document fragments. The Semantic Clipboard addresses these issues by eliminating the reliance of an external document fragment ontology and utilizing the operating system's clipboard to paste the image with the associated license metadata in XHTML. The only requirement for the Semantic Clipboard to work is that the license information about the work must be expressed in RDFa in the source documents.

There are several tools that can be used to automatically embed the license metadata from Flickr. Applications such as ThinkFree, a Web based commercial office suite [27, and the open source counterpart of it, the "Flickr image reuse for OpenOffice.org" [10] are examples of such applications. These applications allow the user to directly pick an image from the Flickr Web site and automatically inject the license metadata with it into a document in the corresponding office 
suite. A severe limitation of this approach is that they only support Flickr images. The Semantic Clipboard can be used to copy any image to any target document along with the license as long as the license metadata is expressed in RDFa.

Attributor [1, a commercial application, claims to continuously monitor the Web for its customers' photos, videos, documents and let them know when their content has been used elsewhere on the Web. It then offers to send notices to the offending Web sites notifying link requests, offers for license, requests for removal or shares of the advertisement revenue from the offending pages. Another commercial application called PicScout [21] claims that it is currently responsible for detecting over $90 \%$ of all online image infringement detections. They also claim to provide the subscribers of their service with a view into where and how their images are being used online. The problem with these services is that they penalize the infringers, rather than encouraging them to do the right thing upfront [16]. In addition to that, since their implementations are based on bots that crawl the Web in search of infringements, these services take up valuable Internet bandwidth [30. Also, these services are not free, which bars many content creators who wish to use such services to find license violations of their content from using the service.

\section{Future Work}

Currently, the images that are copied with their metadata to the Semantic Clipboard are overwritten when some new content is copied to the clipboard. In other words, the tool only supports copying of one image at a time. But it would be useful to have a persistent data storage to register images or any other Web media along with their license metadata, index them, make persistent across browser sessions, and use the copied content whenever the user needs it in a licensecompliant manner. One other main drawback of the Semantic Clipboard is that it is Firefox browser-dependent. Developing an Opera Widget, a Chrome Extension, a Safari Plugin, an Internet Explorer Content Extension or completely making this tool browser independent seems to be a viable future direction of the project.

Also, the tools we have developed only works if every image found on the page has it's own license. Possible extensions would be to have higher level of granularity to determine the license of an image when it does not have a license of it's own, but is contained within a page that has a license or is a member of a set of images (e.g. a photo album) that has a license. The Protocol for Web Description Resources (POWDER) [22], a mechanism that allows the provision of descriptions for groups of online resources, seems like a viable method to making the license descriptions about the resources explicit. Tool builders can then rely on the POWDER descriptions to help users to make appropriate content reuse decisions.

It would be interesting to measure how user behavior changes with the introduction of tools such as the License Violations Validator and the Semantic Clipboard. A measurement of the change in the level of license awareness would 
be an important metric in determining the success of these tools. Therefore, we plan to perform a controlled user study in the future.

We have only explored one domain of content, specifically image reuse on the Web. However, there are billions of videos uploaded on YouTube, and potentially countless number of documents on the Web, which have various types of licenses applied. While organizations such as Mobile Picture Association of America (MPAA), Recording Industry Association of America (RIAA) and other such organizations are working towards preserving the rights of the works of their artists on YouTube, other video and audio sharing sites and peer-to-peer file sharing networks, there are no viable alternatives for ordinary users who intend to protect their rights using CC. Thus a solution of this nature which detects $\mathrm{CC}$ license violations based on the metadata of other types of free-floating Web media will be very useful.

The requirement for attribution is a form of social compensation for reusing one's work. While mentioning one's name, homepage or the WebID when attributing draws attention to an individual, other forms of 'attention mechanisms' can also be implemented. For example, a content creator can obligate the users of her works to give monetary compensation or require that they include certain ad links in the attribution XHTML or give attribution in an entirely arbitrary manner. These extra license conditions can be specified using the cc:morePermissions property. Tools can be built to interpret these conditions and give credit to the original creator as requested.

We envision that the same principle used for checking attribution license violations could be used for checking other types of license violations. Detecting whether an image has been used for any commercial use would be of much interest to content creators, especially if the second use of the image decreases the monetary value of the original image. The CC deed for Non Commercial (NC) use specifies that a license including the $\mathrm{NC}$ term may be used by anyone for any purpose that is not "primarily intended for or directed towards commercial advantage or private monetary compensation". However, this definition can be vague in certain circumstances. Take, for example, the case where someone uses a CC-BY-NC licensed image in her personal blog properly attributing the original content creator. The blog is presumably for non commercial use, and since she has given proper attribution, it appears that no license violation has occurred. However there might be advertisements in the page that are generated as a direct result of the embedded image. Our user might or might not actually generate revenue out of these advertisements. But if she does, it could be interpreted as a 'private monetary compensation'. Hence we believe that the perception as to what constitutes a 'Commercial Use' is very subjective.

$\mathrm{CC}$ recently conducted an online user survey to gather general opinions as to what people perceive a 'Commercial Use' is [6]. An important finding from this survey is that $37 \%$ of the users who make money from their works do so indirectly through advertisements on their Web pages. Therefore, it seems that there aren't any clear cut definitions of a 'Non Commercial Use' yet to find out violations and gather experimental results. But, if the definition of non commercial use 
becomes clearer and much more objective, a validator can be implemented to check for such violations as well. It would also be interesting to check for sharealike license violations. These violations happen when a conflicting license is given when the content is reused. The solution, therefore, is to check the RDFa in both the original page and in the page where the image was embedded to see if the latter is the same as the original CC license.

\section{Conclusion}

As our license violations experiment indicated, there is a strong lack of awareness of licensing terms among content reusers. This raises the question as to whether machine readable licenses are actually working. Perhaps more effort is needed to bring these technologies to the masses, and more tools are needed to bridge the gap between the license-aware and the license-unaware. There should also be methods to find out license violations when users are not cooperative.

An important research question that stems from this work is the method of provenance preservation of content on the Web. We have trivially assumed URIs to be the provenance preservation mechanism when developing the tools described. However, it would be an interesting challenge to track provenance based on the content itself, without having to rely on a unique identifier such as a URI. This would enable us to find out license violators, in addition to validating one's own work for any violations. Also, programmatically determining whether a particular reuse of material is allowable or not is subjective, especially since some of the laws and standards have been quite ambiguous in defining these terms.

In general, social constraints are functions of any part of the blossoming Social Web we are experiencing today. As we are living in an era of increasing user generated content, these constraints can be used to communicate the acceptable uses of such content. We need tools, techniques and standards that strike an appropriate balance between the rights of the originator and the power of reuse. The rights of the originator can be preserved by expressing what constitutes appropriate uses and restrictions using a rights expression language. These rights will be both machine and human readable. Reuse can be simplified by providing the necessary tools that leverage these machine readable rights to make the users more aware of the license options available and ensure that the user be license or policy compliant. Such techniques can be incorporated in existing content publishing platforms or validators or even Web servers to make the process seamless. This paper has demonstrated several tools that enable the development of such policy-aware systems, and we hope that these will stimulate research in this area in the future.

\section{Acknowledgements}

Parts of this work was done while the first author was undergoing the 'Networks for Web Science Research Exchange' program under the guidance of Nigel 
Shadbolt at University of Southampton, UK. Special thanks for his advice all throughout the project. In addition, the authors wish to thank their colleagues, Danny Weitzner, Hal Abelson, Gerry Sussman, and other members at DIG for their contribution to the ideas expressed in this paper.

This work was supported by NSF Cybertrust award CT-M: 0831442, IARPA award FA8750-07-2-0031, and UK Engineering and Physical Sciences Research Council (EPSRC) grant EP/F013604/.

\section{References}

1. Attributor - Subscription based web monitoring platform for content reuse detection, http://www. attributor.com

2. blip.tv - Hosting, distribution and advertising platform for creators of web shows

3. Bonatti, P.A., Duma, C., Fuchs, N., Nejdl, W., Olmedilla, D., Peer, J., Shahmehri, N.: Semantic web policies - a discussion of requirements and research issues. In: Sure, Y., Domingue, J. (eds.) ESWC 2006. LNCS, vol. 4011, pp. 712-724. Springer, Heidelberg (2006)

4. Creative Commons BY 3.0 Unported Legal Code, http://creativecommons.org/licenses/by/3.0/legalcode

5. Creative Commons Customized Search in Google, http://creativecommons.org/press-releases/entry/5692

6. Creative Commons Noncommercial study interim report,http://mirrors . creativecommons . org/nc-study/NC-Use-Study-Interim-Report-20090501.pdf

7. Exchangeable Image File Format, http://www.exif.org/specifications.html

8. Feigenbaum, J., Freedman, M.J., Sander, T., Shostack, A.: Privacy engineering for digital rights management systems. In: Sander, T. (ed.) DRM 2001. LNCS, vol. 2320, pp. 76-105. Springer, Heidelberg (2002)

9. Flickr API, http://www.flickr.com/services/api

10. Flickr image reuse for openoffice.org, http://wiki.creativecommons.org/ Flickr-Image-Re-Use-for-OpenOffice.org

11. Abelson, H., Adida, B., Linksvayer, M., Yergler, N.: ccREL: The Creative Commons Rights Expression Language. Creative Commons Wiki (2008)

12. How to attribute Flickr images, http://www. squidoo.com/cc-flickr/\#module12311035

13. Dworak, H.: Creative Commons License Validation Service, http://validator . creativecommons . org

14. International Press Telecommunications Council Photo Metadata Format, http://www.iptc.org/IPTC4XMP

15. Jones, H.C.: Xhtml documents with inline, policy-aware provenance. Master's thesis, Massachusetts Institute of Technology (May 2007)

16. Doctor, K.: Blog Entry on Attributor Fair Syndication Consortium Completes Newspaper Trifecta, http://www.contentbridges.com/2009/04/ attributor-ad-push-on-piracy-completes-newspaper-trifecta.html

17. Kim, J.W., Candan, K.S., Tatemura, J.: Efficient overlap and content reuse detection in blogs and online news articles. In: 18th International World Wide Web Conference WWW 2009 (April 2009)

18. Kishor, P., Seneviratne, O.: Public policy: Mashing-up technology and law. In: Mashing-up Culture: The Rise of User-generated Content, COUNTER workshop, Uppsala University (May 2009) 
19. MozCC - Firefox extension to discover Creative Commons licenses, http://wiki.creativecommons . org/MozCC

20. OWL Music Search, http://www.owlmusicsearch.com

21. picScout - Image tracker for stock photography agencies and professional photographers, http://www.picscout.com

22. Protocol for Web Description Resources (POWDER), http://www.w3.org/2007/powder

23. RDF, Resource Description Framework, http://www.w3.org/TR/rdf-syntax-grammar

24. RDFa, Resource Description Framework in Attributes, http: //www. w3.org/2006/07/SWD/RDFa/syntax

25. Shivakumar, N., Garcia-Molina, H.: Scam: A copy detection mechanism for digital documents. In: Second Annual Conference on the Theory and Practice of Digital Libraries (1995)

26. SpinXpress - Collaborative media production platform, http://spinxpress.com

27. Think Free - Java based web office suite, http://www. thinkfree.com

28. Berners-Lee, T., Hollenbach, J., Lu, K., Presbrey, J., Prud'ommeaux, E., Schraefel, M.C.: Tabulator Redux: Browing and Writing Linked Data. In: Linked Data on the Web Workshop at WWW (2008)

29. Weitzner, D.J., Abelson, H., Berners-Lee, T., Feigenbaum, J., Hendler, J., Sussman, G.J.: Information accountability. Communications of the ACM (June 2008)

30. Faulkner, W.: Tales From The IT Side: PicScout, Getty Images and Goodbye iStockPhoto..!,

http://williamfaulkner.co.uk/wordpress/2007/09/ picscout-getty-images-and-goodbye-istockphoto

31. XMP - Extensible Metadata Platform, http://www . adobe.com/products/xmp/index.html

32. Yahoo Creative Commons Search, http://search. yahoo.com/cc 